\title{
Grüne, Anne/Hafez, Kai/Priyadharma, Subekti/Schmidt, Sabrina (Hrsg.): Media and transformation in Germany and Indonesia: asymmetrical comparisons and perspectives
}

\author{
Berlin: Frank \& Timme 2019. 346 Seiten. Preis: $€ 59,80$
}

\section{Ines Drefs}

Online publiziert: 29. Juni 2020

(C) Der/die Autor(en) 2020

Selbst die vergleichende Mediensystemforschung verbleibt allzu oft in der sogenannten ,westlichen Welt“. Der vorliegende Tagungsband zu der im November 2017 durchgeführten Konferenz „Media and Transformation in Germany and Indonesia: Dynamics and Regressions in Global Perspective“ verspricht dagegen neuartige Perspektiven und Erkenntnisse, die sich aus dem Vergleich zweier kulturell, politisch und ökonomisch sehr unterschiedlicher Länder speisen.

Gegliedert ist das Buch in vier Teile. Der erste Teil mit dem Titel „Media and Political Transformation" beginnt mit einer vergleichenden Arbeit von Mira RochyadiReetz und Martin Löffelholz. Dabei handelt es sich um eine Gegenüberstellung der Mediensysteme Indonesiens und Deutschlands, die sowohl etablierte Indikatoren (,media markets“, „,political parallelism“, ,,journalistic professionalism“, ,state intervention“) als auch neuere Indikatoren (,press freedom“, ,,internet freedom“, ,media trust") aus der vergleichenden Mediensystemforschung berücksichtigt und diese anhand von Sekundärdaten beleuchtet. Es folgen zwei Aufsätze, die sich jeweils dem Mediensystem Indonesiens und dem Mediensystem Deutschlands widmen und zwar mit unterschiedlicher Schwerpunktsetzung: Während Ade Armando einen historischen Abriss zum Verhältnis von Staat und Medien in Indonesien seit 1945 liefert, fokussieren Oliver Hahn und David Liewehr ihre Analyse auf die politische Kommunikation rund um die deutsche Bundestagswahl im Jahr 2017.

Der zweite Teil ist dem Thema „Media Representation and Racism“ gewidmet und startet mit einem theoretischen Beitrag von Sabrina Schmidt. Die Autorin schlägt darin eine Verknüpfung von Rassismustheorie und normativen Öffentlichkeitsmodellen von Habermas und Husband vor, mit dem Ziel, einen Analyserahmen zu

Dr. I. Drefs ( $\square)$

Erich-Brost-Institut für internationalen Journalismus, Technische Universität Dortmund, Otto-Hahn-Str. 2, 44227 Dortmund, Deutschland

E-Mail: ines.drefs@tu-dortmund.de 
schaffen, der transnationale Vergleiche jenseits von Eurozentrismus ermöglicht. Im anschließenden Beitrag stützt sich Ratna Noviani auf konzeptionelle Überlegungen $\mathrm{zu}$,representation“, um anhand vier zeitgenössischer indonesischer Filme zu untersuchen, ob die gesteigerte mediale Sichtbarkeit der chinesisch-indonesischen Minderheit des Landes mit einer differenzierteren Darstellung einhergeht. Margreth Lünenborg weist im letzten Beitrag dieses Teils auf die besondere Relevanz des Konzepts der ,cultural citizenship“ in einer globalisierten Welt hin. Ob deutsche Printmedien geeignete Bedingungen dafür schaffen, untersucht sie am Beispiel visueller Darstellungen von migrationsbezogenen Themen zwischen 2006 und 2015.

Der dritte Teil trägt den Titel „Internet and Counter Public“. Subekti Priyadharma stellt im ersten Beitrag die Internet-Öffentlichkeiten Indonesiens und Deutschlands gegenüber. Dabei orientiert er sich an fünf Dimensionen: Zugang zu Informationen, Wechselseitigkeit der Kommunikation, Kommerzialisierung, ethische Aspekte und rechtliche Aspekte. Im darauffolgenden Beitrag befasst sich Jeffrey Wimmer - ohne spezifischen Länderfokus - mit Bedingungen und Charakteristika von Gegenöffentlichkeiten, alternativen Medien und Partizipation vor dem Hintergrund der Digitalisierung.

Der vierte Teil firmiert unter dem Titel „Popular Culture and Democracy“ und enthält neben spezifischen Beobachtungen zu Deutschland und Indonesien auch eine ägyptische Perspektive. Diese wird von Randa Aboubakr eingebracht, die in ihrem Beitrag darlegt, wie der vormals staatlich kontrollierte öffentliche Raum nach Absetzung Mubaraks mittels populärkultureller Erzeugnisse (zumindest zeitweise) im Sinne einer modernen Agora zurückerobert wurde. Partizipatorische Aspekte populärkultureller Produktion greift auch Anne Grüne im darauffolgenden Beitrag auf. Anhand indonesischer und deutscher Beispiele arbeitet die Autorin den letztendlich ambivalenten Charakter von Populärkultur in verschiedenen Perioden politischer Verfasstheit heraus: Je nach Transformationsphase kann sie, so Grünes These, demokratiefördernd oder auch demokratieschädigend wirken. Auf die eher destruktive Rolle von Populärkultur innerhalb des gegenwärtigen politischen Systems Indonesiens geht Yasraf A. Piliang ein. In seinem Beitrag stellt er fest, dass populärkulturelle Erzeugnisse insbesondere nach der Umwandlung des Wahlsystems von einem indirekten zu einem direkten zunehmend politisch instrumentalisiert werden. Er erkennt darin eine individualistisch-liberale Tendenz, die im Gegensatz steht zur gemeinschafts- und konsensbetonten Staatsdoktrin „Pancasila“, in der sich Merkmale eines deliberativen Demokratiemodells widerspiegeln.

Die Herausgeber des Bandes verwenden in der Einleitung recht viel Energie darauf, den vermeintlich ,,asymmetrischen“ Vergleich zwischen Deutschland und Indonesien zu rechtfertigen und dem antizipierten Vorwurf zu begegnen, sie würden Äpfel mit Birnen vergleichen. Dabei ist ein solcher Vergleich im Sinn eines ,,most different systems design“" vollkommen legitim - wenn nur klar wird, welche Vergleichsdimensionen unter welchen Annahmen betrachtet werden. Im Band werden zwar immer wieder Gemeinsamkeiten zwischen Deutschland und Indonesien, wie z.B. das historische Erbe eines Genozids oder gegenwärtige Radikalisierungstendenzen angeführt, es werden daraus jedoch weder Annahmen noch Analyseschwerpunkte abgeleitet. So bleibt es insgesamt bei eher deskriptiven Gegenüberstellungen als bei aussagekräftigen Vergleichen. Versteht man den Band eher als explorativen 
Vorstoß, so stellt er sich aber als äußerst reichhaltig dar. Er enthält vielversprechende theoretische Ansätze, die sich in Zukunft für einen einheitlichen Analyserahmen vergleichender Forschung anböten. Außerdem könnten die dargelegten Gemeinsamkeiten und Unterschiede zukünftigen Forschungsvorhaben als Variablen dienen, auf deren Basis sich der Erkenntnisgewinn um Kausalerklärungen erweitern ließe.

Funding Open Access funding provided by Projekt DEAL.

Open Access Dieser Artikel wird unter der Creative Commons Namensnennung 4.0 International Lizenz veröffentlicht, welche die Nutzung, Vervielfältigung, Bearbeitung, Verbreitung und Wiedergabe in jeglichem Medium und Format erlaubt, sofern Sie den/die ursprünglichen Autor(en) und die Quelle ordnungsgemäß nennen, einen Link zur Creative Commons Lizenz beifügen und angeben, ob Änderungen vorgenommen wurden.

Die in diesem Artikel enthaltenen Bilder und sonstiges Drittmaterial unterliegen ebenfalls der genannten Creative Commons Lizenz, sofern sich aus der Abbildungslegende nichts anderes ergibt. Sofern das betreffende Material nicht unter der genannten Creative Commons Lizenz steht und die betreffende Handlung nicht nach gesetzlichen Vorschriften erlaubt ist, ist für die oben aufgeführten Weiterverwendungen des Materials die Einwilligung des jeweiligen Rechteinhabers einzuholen.

Weitere Details zur Lizenz entnehmen Sie bitte der Lizenzinformation auf http://creativecommons.org/ licenses/by/4.0/deed.de.

Dr. Ines Drefs ist Postdoktorandin am Erich-Brost-Institut für internationalen Journalismus der Technischen Universität Dortmund. 